\title{
Measurement of the Drift Velocity of Holes in Silicon at High-Field Strengths
}

\author{
V. RODRIGUEZ, H. RUEGG, MEMber, IEEE, AND M-A. NICOLET, MEMber, IEEE
}

\begin{abstract}
A method is presented which allows the measurement of the velocity-field relationship of charge carriers in a semiconductor. The device used is a four-layer structure. The mode of operation is based on the injection by punch-through of charge carriers into a long depleted region. The velocity can be determined from the V-I characteristic of the device and its geometry. Drift velocity saturation is indicated directly by the form of the characteristic.

The method has been applied to the measurement of the highfield velocity of holes in silicon. Technological limitations restricted the measurements to fields above $4 \cdot 10^{4} \mathrm{~V} / \mathrm{cm}$. From this value up to $11 \cdot 10^{4} \mathrm{~V} / \mathrm{cm}$ the hole velocity is found to be constant and equal to $7.5 \cdot 10^{5} \mathrm{~cm} / \mathrm{s} \pm 5 \%$.
\end{abstract}

\section{INTRODUCTION}

$\mathrm{T}$ IHE FIRST EXPERIMENTS which show that in germanium and silicon the drift velocity of mobile charge carriers is no longer proportional to the electric field $E$ at high-field strengths were performed by Ryder [1]. Shockley [2] interpreted the results in terms of lattice scattering and identified three ranges: a) the linear (ohmic) range at low fields, b) a transition range in which the velocity increases as $E^{1 / 2}$, and c) a saturation range in which the drift velocity is constant, $v_{\varepsilon}$, and independent of $E$ for fields larger than the saturation field $E_{s}$. Prior [3], using somewhat different experimental techniques, confirmed the departures from linearity described by Ryder, but was unable to identify a transition range in which the drift velocity would increase as $E^{1 / 2}$ over any significant range and the existence of a saturation velocity remained questionable. Moreover, his mobility values change unduly with the resistivity of the samples, an effect indicating the probable presence of carrier injection at the contacts.

Interest in the behavior of the drift velocity at highfield strengths has risen again in recent years, motivated partly by new theoretical analyses of the carrier-lattice interactions of charge carriers in a solid [4], as well as by a number of fast solid-state devices operating at very large internal field strengths [5]-[7]. The present study was therefore undertaken with an aim at supplying more accurate experimental data on the subject. The difficulty encountered by Prior with carrier injection at the contacts was circumvented in our approach

Manuscript received October 27, 1966. The work reported was performed at Fairchild Semiconductor in the course of a summer employment of $\mathrm{V}$. Rodriguez

H. Ruegg is with Fairchild Semiconductor, Palo Alto, Calif.

V. Rodriguez and M-A. Nicolet are with the Department of Electrical Engineering, California Institute of Technology, Pasadena, Calif. by making use of it, rather than trying to suppress it. Experiments were performed in silicon because of its dominating practical significance, using holes as the mobile charge carriers.

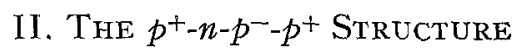

The device used for the measurements is a four-layer $p^{+}-n-p^{-}-p^{+}$structure of planar goemetry [see Fig. 1(a)]. The respective widths of the layers are typically $4 \mu, 1 \mu, 15 \mu$ and, approximately, $100 \mu$, the latter being the thickness of the original substrate on which the structure is fabricated. A potential is applied such as to reverse bias the $n-p^{-}$junction. The resulting distribution for the electric field is depicted in Fig. 1(b). At the smallest bias shown, the structure is essentially equivalent to a junction transistor operated with a floating base, the narrow $n$-region representing the base and the $p^{-}-p^{+}$regions being the collector. The wide and lowly doped $p^{-}$section of this collector generates a region of nearly uniform electric field. The much more heavily doped adjacent $n$ and $p^{+}$regions, on the other hand, cause a very rapid falloff of the field within distances much shorter than the width of the $p$ region. Consequently, a hole drifting through this structure will encounter a nearly constant field. Holes are now injected into the base by raising the applied potential above the punch-through voltage $V_{p t}$. These holes being injected into a depleted region will generate an additional electric field by virtue of their space charge. Their distribution will depend upon the relationship existing between the drift velocity and the electric field.

If this relationship is such that the drift velocity saturates and if, at punch-through, this electric field exceeds the saturation value $E_{s}$ throughout the $p^{-}$region and most of the base, the density of the injected holes will be spacially uniform and equal to $j / v_{s}$. This insures a linear relationship between the current and the potential $V^{\prime}=V-V_{p t}$ above punch-through since a two-fold integration of Poisson's equation yields

$$
j=2 \epsilon \vartheta_{s} V^{\prime} /\left(w+x_{0}\right)^{2}
$$

where $w$ is the width of the $p^{-}$region and $x_{0}$ is that of the base and where $\epsilon$ is the dielectric constant of silicon. The accuracy of this equation will increase with increasing values of the punch-through potential. The resulting V-I characteristic is shown in Fig. 2.

If, at punch-through, the field does not exceed $E_{s}$ everywhere or if a limiting drift velocity does not exist, 
the distribution of the injected holes will no longer be uniform. The additional electric field generated by their space charge will, however, always tend to compensate that of the ionized acceptors in the $p^{-}$region. In particular, an operating point will exist at which the electric field in that region is approximately uniform. Under such conditions a measurement of the incremental changes $\Delta V$ and $\Delta j$ of the voltage and the current density should yield the drift velocity $v(\bar{E})$ at the average field value $\bar{E}$ in the $p^{-}$region. An argument similar to that of above then suggests that

$$
\Delta j=2 \epsilon v(\bar{E}) \Delta V /\left(w+x_{0}\right)^{2}
$$

should hold. The error in this approximation may be significant due to the uncertainty in the contribution to the electric field of the holes injected in the base. It should thus be possible with structures of this type to obtain the velocity-field relationship below $E_{s}$, but detailed analytical studies will have to be conducted to exploit the method in that range.

The onset of avalanche multiplication limits the field range in which this technique is applicable. The above description has been given in terms of holes. The method can of course be adapted to electrons by using a $n^{+}-p-n^{-}-n^{+}$structure.

The present method may be compared to similar experiments using an $n^{+}-p^{-}-n^{+}$structure as described by Denda and Nicolet [7]. Since in their device the field at the emitter is zero and gradually rises toward the collector, it is only possible to infer the existence of a saturating velocity and to estimate its value from the asymptotic behavior of the $\mathrm{V}-\mathrm{I}$ characteristic. The use of a four-layer structure can be regarded as an extension of the method described by Denda and Nicolet whereby the center region is divided into a short portion with a very steep field rise and a long portion with a substantially constant field.

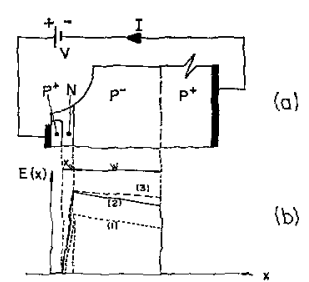

Fig. 1. The $p^{+}-n-p^{-}-p^{+}$structure (a) and the field distribution (b) in the $p^{-}$region for an applied voltage smaller than (1), equal to (2) and larger than (3) the punch-through voltage.

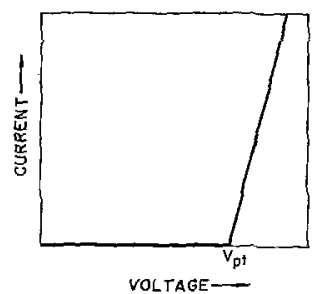

Fig. 2. Idealized V-I characteristic for the case that the electric field strength in the depleted region exceeds $E_{\text {s a }}$ at punch-through.

\section{Measurement of the Velocity of HOLES IN SILICON}

The technique described above has been applied to measure the saturation velocity of holes in silicon. Figure 1 (a) shows a cross section of the epitaxial mesa devices used. A $15 \mu$ thick $p$-type layer with a resistivity of roughly $10^{2} \Omega-\mathrm{cm}$ is first grown on a low resistivity $p^{+}$type substrate. A $5 \mu$ thick layer of $1 \Omega$-cm $n$-type material is deposited next. Using standard photolithographic and oxide masking techniques, circular emitters with a diameter of 0.004 inch are diffused in to the $n$-type layer. After the etching of mesas with a diameter of 0.006 inch, the diffusion of the emitters is continued in small time increments while the progressive decrease of the punchthrough voltage is monitored. In this way batches of devices are obtained which are identical in every respect except for different base widths and hence different punch-through voltages $V_{p t}$.

On some devices measurements were performed with a point contact after the final emitter diffusion, while others were provided with an evaporated and alloyed aluminum contact to the emitter region and mounted into standard transistor packages for further evaluation. Measurements were performed with a curve tracer. The absence of excessive internal heating in the devices was checked by comparing these characteristics with those obtained from pulse measurements.

A typical set of characteristics obtained from devices

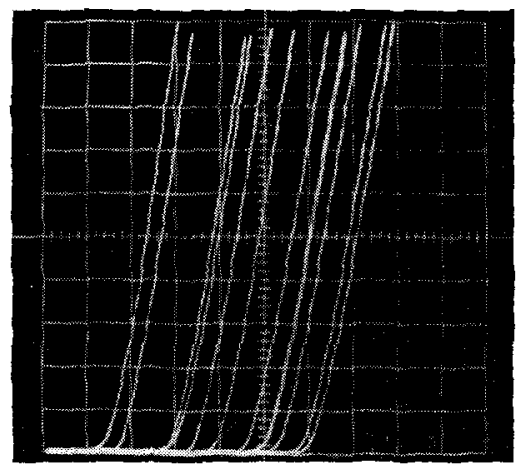

Fig. 3. Experimental V-I characteristics from a set of $p^{+}-n-p^{-}-p^{+}$ devices of different base width. Scales: vertical $2 \mathrm{~mA} / \mathrm{cm}$, horizontal $20 \mathrm{~V} / \mathrm{cm}$.

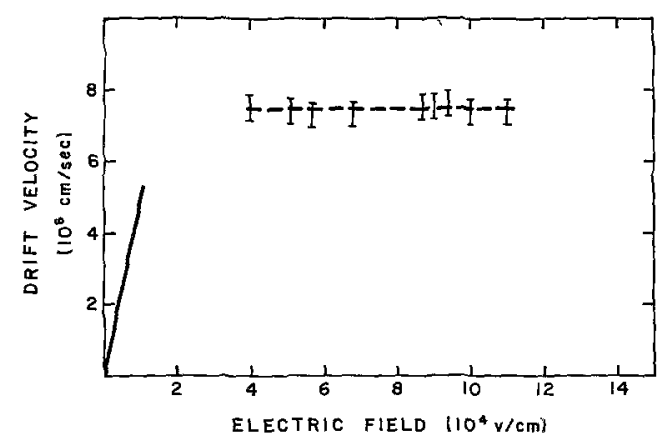

Fig. 4. Experimental results. The solid line corresponds to the low-field mobility obtained by Ryder [1]. 
of different base widths is shown in Fig. 3. Except for the variation in the punch-through voltage $V_{p t}$ the characteristics are observed to be essentially the same. Beyond punch-through, this characteristic is linear. Both facts indicate that the holes indeed move with constant drift velocity at the field strengths attained. They range from 4-11.104 V/cm. A typical analysis of such characteristics based on (1) is shown in Fig. 4. The result obtained on approximately 100 separate devices yields a value of $7.5 \cdot 10^{6} \mathrm{~cm} / \mathrm{s}$ with an estimated error of \pm 5 percent for the saturation velocity of holes in silicon at room temperature. The values of $\left(w+x_{0}\right)$ were obtained either from cross-section measurements or from capacitance measurements at a bias voltage slightly below punch-through.

\section{CONCLUSION}

The results presented above demonstrate that the drift velocity of holes in silicon saturates at room temperature. They also establish the advantage of using rather than suppressing injection in studying the behavior of charge carriers at high-field strengths. This technique is capable of yielding results also in the absence of velocity saturation. In addition, it is readily adaptable to a large range of ambient temperatures. It is also applicable equally to holes and electrons. The main limitations-aside from ionization effects-resides in the requirement of an established technology to manufacture junction devices. This presently restricts the method to a limited number of materials.

\section{REFERENCES}

[1] E. J. Ryder, "Mobility of holes and electrons in high electric fields,"Phys. Rev., vol. 90, pp. 766-769, June 1953.

[2] W. Shockley, "Hot electrons in germanium and ohm's law," Beil Sys. Tech. J., vol. 30, pp. 990-1034, October 1951.

[3] A. C. Prior, "The filed-dependence of carrier mobility in silicon and germanium," J. Phys. Chem. Solids, vol. 12, pp. 175-180, 1959.

[4] K. K. Thornber, to be published.

[5] K. M. Johnson, "High-speed photodiode signal enhancement at avalanche breakdown," IEEE Trans. on Electron Devices, vol. ED-12, pp. 55-63, February 1965.

[6] B. C. De Loach and R. L. Johnston, "Avalanche transit-time microwave oscillators and amplifiers," IEEE Trans. on Electron Derices, vol. ED-13, pp. 181-186, January 1966.

[7] S. Denda and M-A. Nicolet, "Pure space-charge-limited electron current in silicon," J. Appl. Phys., vol. 37, pp. 2412-2424, May 1966.

\title{
Electron Drift Velocity in Avalanching Silicon Diodes
}

\author{
C. Y. DUH, MEMber, IEEE AND J. L. MOLL, FeLlow, IEEE
}

\begin{abstract}
The differential resistance of an avalanching $p^{+} n n^{+}$ junction is used to obtain the electron drift velocity at electric fields where significant avalanching is occurring $\left(2 \times 10^{5}<E<4 \times 10^{5}\right.$ $\mathrm{V} / \mathrm{cm}$ ). The velocity is also obtained as a function of temperature and is consistent with energetic phonon scattering.
\end{abstract}

\section{INTRODUCTION}

S HOCKLEY has suggested the measurement of space-charge conductance in avalanching $p-n$ junctions to obtain carrier drift velocity in depletion regions. Rodriquez and Ruegg [1] have used spacecharge conductance of space-charge limited current in $p^{+} n p^{-} p^{+}$diodes to obtain hole drift velocity. As [1] shows, the space-charge conductance of a drift space of wid th $W_{d}$ is

$$
\sigma=\frac{\Delta I}{\Delta V}=\frac{2 \epsilon v(E) A}{W_{d^{2}}^{2}}
$$

where the symbols have their usual meaning.

Manuscript received November 21, 1966

The authors are with Stanford University, Stanford, Calif.
Equation (1) is generally true if the electric field at the plane of injection is constant, independent of $\Delta I$. A typical avalanche diode does not satisfy these conditions. This paper will extend the theory of space-charge conductance to typical $p^{+} n n^{+}$diodes and present measurements of space-charge conductance and finally electron drift velocity.

\section{Avalanche Space-Charge Conductance: $p^{+} n n^{+}$DIODES}

It is desirable to relate the space-charge conductance of avalanching diodes to geometrical and material parameters which include carrier drift velocity. This process is extremely difficult to accomplish without making a linearized model for the ionizing region of the diode. The genesis of the linearized model may be traced from Figs. 1 and 2. Figure 1 shows the electric field encountered in a $p^{+} n n^{+}$junction. Most of the ionization occurs near the $p^{+} n$ junction where the field is a maximum. 